\title{
SIFT Texture Description for Understanding Breast Ultrasound Images
}

\author{
Joan Massich ${ }^{12, \star}$, Fabrice Meriaudeau ${ }^{2}$, Melcior Sentís ${ }^{3}$, Sergi Ganau ${ }^{3}$, \\ Elsa Pérez ${ }^{4}$, Domenec Puig ${ }^{5}$, Robert Martí ${ }^{1}$, Arnau Oliver ${ }^{1}$, and Joan Martí ${ }^{1}$ \\ 1 Computer Vision and Robotics Group, University of Girona, Spain \\ jmassich@atc.udg.edu \\ 2 Laboratoire Le2i-UMR CNRS, University of Burgundy, Le Creusot, France \\ 3 Department of Breast and Gynecological Radiology, UDIAT-Diagnostic Center, \\ Parc Taulí Corporation, Sabadell, Spain \\ ${ }^{4}$ Department of Radiology, Hospital Josep Trueta of Girona, Spain \\ 5 Department of Computer Engineering and Mathematics, University Rovira i \\ Virgili, Tarragona, Spain
}

\begin{abstract}
Texture is a powerful cue for describing structures that show a high degree of similarity in their image intensity patterns. This paper describes the use of Self-Invariant Feature Transform (SIFT), both as low-level and high-level descriptors, applied to differentiate the tissues present in breast US images. For the low-level texture descriptors case, SIFT descriptors are extracted from a regular grid. The high-level texture descriptor is build as a Bag-of-Features (BoF) of SIFT descriptors. Experimental results are provided showing the validity of the proposed approach for describing the tissues in breast US images.
\end{abstract}

Keywords: breast cancer, ultrasound, texture, SIFT.

\section{Introduction}

Breast cancer is the second most common cancer (1.4 million cases per year, $10.9 \%$ of diagnosed cancers) after lung cancer, followed by colorectal, stomach, prostate and liver cancers. In terms of mortality, breast cancer is the fifth most common cause of cancer death. However, it places as the leading cause of cancer death among females both in western countries and in economically developing countries 3 .

Medical imaging plays an important role in breast cancer mortality reduction, contributing to its early detection through screening, diagnosis, image-guided biopsy, treatment follow-up and suchlike procedures [5]. Despite Digital Mammography (DM) still remains as the image modality of reference for diagnose purposes, Ultra-Sound (US) offers useful complementary diagnose information due to its capabilities for differentiating between solid lesions that are benign or

\footnotetext{
* This work was partially supported by the Spanish Science and Innovation grant nb. TIN2012-37171-C02-01 and TTIN2012-37171-C02-02 and the Regional Council of Burgundy.
} 
malignant 6]. It is estimated that between $65 \sim 85 \%$ of the biopsies prescribed using only mammography imaging could be avoided if US information had been taken into account while issuing the diagnose [7.

In US images, texture is a major characteristic to distinguish between different breast tissues, which also allows assessing of the lesion's pathology [6]. Thus, the importance of incorporating texture data from US images into Computer Aided Diagnosis (CAD) systems. A comprehensive list of texture descriptors used for detection, segmentation or diagnose tasks applied to US breast images is given in Cheng et al. [1, where most of the descriptors are ad-hoc descriptors or based on well-known texture descriptors such as co-occurrence matrices, wavelet coefficients or Gray-Level Difference Method (GLDM).

This article explores the usage of Self-Invariant Feature Transform (SIFT) descriptors for encoding the US characteristic texture produced by the speckle noise present within the images. Its performance is evaluated using a multi-label annotated dataset.

\section{Material and Methods}

In order to develop segmentation methodologies applied to delineate breast lesions in US data, a set of 700 US images was acquired at the UDIAT Diagnostic Centre of Parc Tauli in Sabadell (Catalunya), between 2010 and 2012. All the images were provided with accompanying Ground Truth (GT) delineation of the lesions present in the image. From this image database, a reduced dataset of 16 images corresponding to different patients was selected and complemented with multi-label GT in order to evaluate the texture description of the observable tissues in the breast.

Figure 1 illustrates a breast image from the dataset with its associated GT.

\section{Using SIFT as a Low-level Texture Descriptor in Order to Differentiate the Tissues Present in Breast US Images}

Self-Invariant Feature Transform (SIFT) 4 transforms key-points into scale and rotation invariant coordinates relative to local features. The SIFT descriptor at
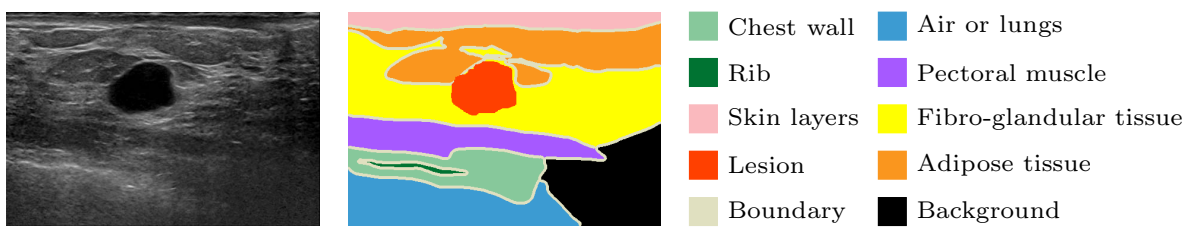

Fig. 1. Dataset sample. From left to right: image sample, accompanying multi-label GT, tissue label GT color-coding. 


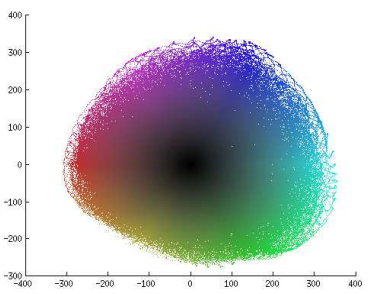

(a)

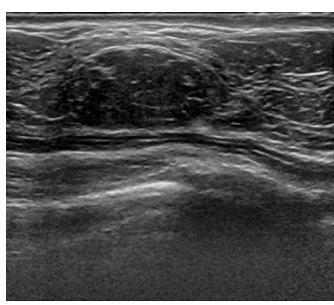

(b)

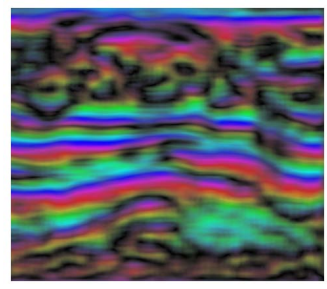

(c)

Fig. 2. Low level SIFT descriptor example. (a) Arbitrary coloring of the projected SIFT space. (b) Original image. (c) Recoding of the extracted SIFT descriptors using the color coding in (a).

a particular key-point, samples the magnitude and orientation of the gradients surrounding this key-point to generate a 128-element feature. When setting up SIFT as a texture descriptor, the key-points are considered to be a regular grid in order to generate evenly sparse SIFT descriptors.

The usage of SIFT descriptor brings invariability to scale, rotation and minor affine transformations along with robustness to illumination changes [4, which allows to characterize the tissues despite the variability from US acquisition.

In order to analyze the US images, a SIFT descriptor is extracted at every pixel position and them mapped into the SIFT space. The 128-dimension feature is projected into a two dimensional space using Principal Component Analysis (PCA). When combining features using PCA is convenient to know the ratio known as explained variation, which in this case is given by $\frac{\lambda_{1}+\lambda_{2}}{\sum_{i=1}^{128} \lambda_{i}}=21.6 \%$. For the remaining of the article all the calculations are carried out directly in the projected space. However, it should be assumed that in a higher space with greater explained variation, better separability could be achieved. Figure 2 offers a visual interpretation of a breast US image in terms of low-level SIFT descriptors, where the extracted SIFT descriptors from all the images in the dataset have been projected into the 2D principal component space (Figure2 $2 \mathrm{~h}$ ). These SIFT descriptors have been arbitrary colored in order to visually assess the descriptors (the more similar the colors, the closer the SIFT descriptors).

Thus, the analysis of the tissue distribution is performed in the texture space defined by the SIFT descriptors by means of the Maximum A Posteriori (MAP) estimator, as described in equation 1 .

$$
P\left(\omega \mid \bar{x}_{a}\right)=\frac{P\left(\bar{x}_{a} \mid \omega\right) \cdot P(\omega)}{P\left(\bar{x}_{a}\right)}
$$

Where $P\left(\omega \mid \bar{x}_{a}\right)$ is the probability that the sample $a$ belongs to class $\omega \in W$ (see fig.1b as a reminder of the GT available classes) where $\bar{x}_{a}$ is the feature vector representing the sample $a$, such that $x_{a}^{i}$ is the $i$ th feature. $P\left(\bar{x}_{a} \mid \omega\right)$ corresponds to the Maximum Likelihood (ML) of the feature distribution for a particular class $\omega$, while $P(\omega)$ and $P\left(\bar{x}_{a}\right)$ are the priors for the class and feature respectively. 


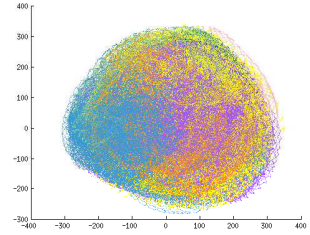

(a)

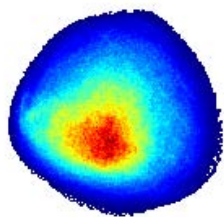

(b)

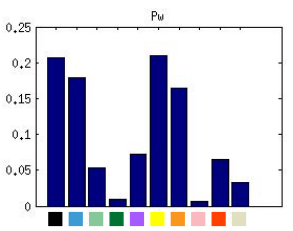

(c)

Fig. 3. SIFT space. (a) Projected space colored according to GT tissue labeling. (b) $P\left(\bar{x}_{a}\right)$. (c) $P(\omega)$

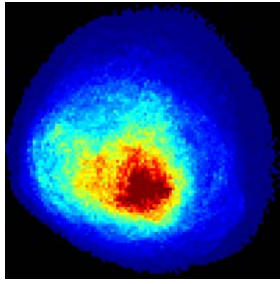

Background

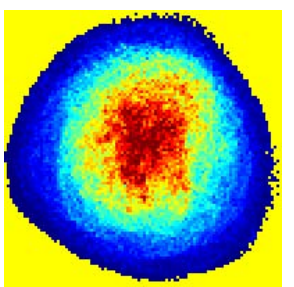

Fibro-glandular

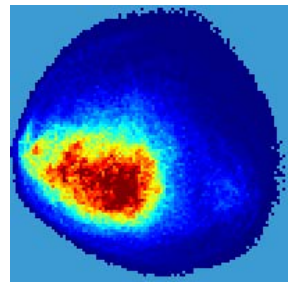

Air or lungs

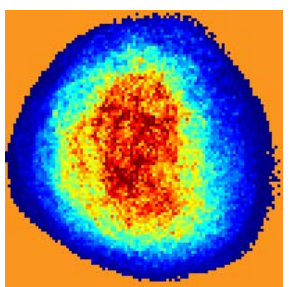

Adipose tissue

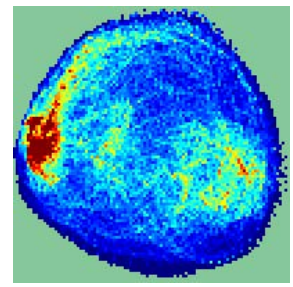

Chest wall

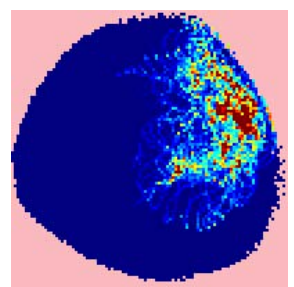

Skin layers

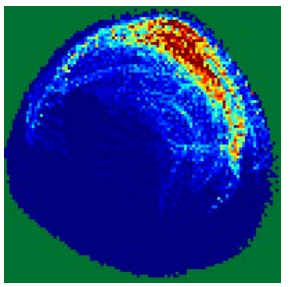

Rib

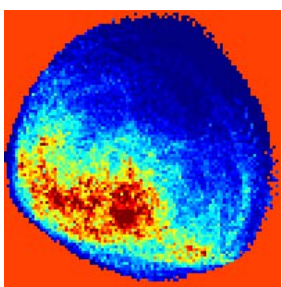

Lesion

Fig. 4. Distribution of the SIFT descriptors for some classes in the GT

Figure 3 uses the entire dataset to illustrate the underlying problem and the priors extracted from the same dataset. Fig. 3 a shows a scatter plot where every sample has been colored according to its GT. Fig. 3b shows an occurrence study of the samples carried out in a discretization of the SIFT space. Fig. 3. illustrates the class prior $P(\omega)$ corresponding to the proportion of samples present in the dataset for each class.

Figure 4 shows the feature distribution study for every class, corresponding to the $P\left(\bar{x}_{a} \mid \omega\right)$ in eq. 1. Similarities and dissimilarities between classes can be observed through the tendencies within the features representing each class. To illustrate that, it can be observed in figure 1 that the adipose tissue class contains Cooper's ligaments which are highly dense fibers, and fibro-glandular tissue is made of dense fibers and unstructured fat. Or, the difficulty to produce accurate GT delineations, which often happens for those regions where the structures are not clear enough to the user (i.e. the background class in fig.(1). 


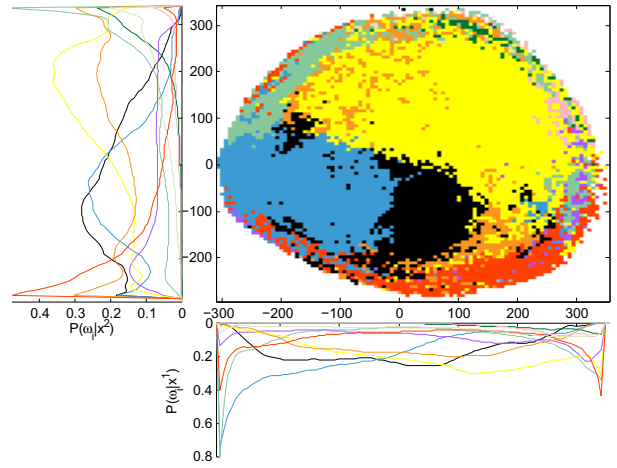

(a) SIFT

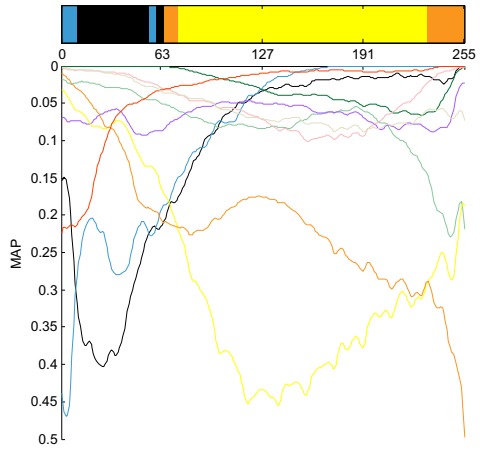

(b) Intensity

Fig. 5. Qualitative evaluation of the MAP labeling of the feature space

Equation 2 illustrates how to produce the preferred labeling of the space, as is illustrated in fig. [5a. On it, the marginals $P\left(\omega_{i} \mid x^{j}\right)$ where $j \in\{1,2\}$ are also represented to obtain a deeper understanding of the MAP.

$$
\text { labeling }(\bar{x})=\underset{i}{\arg \max } P\left(\omega_{i} \mid \bar{x}\right) \quad \text { where } i \in[1 . .|W|]
$$

For comparison purposes, the labeling process has been carried out on the SIFT space as well as on the intensity space to analyze the tissue characterization. Figure 5 shows the qualitative evaluation of the MAP labeling for both spaces. From this results, the SIFT feature space is preferred since when using intensity some of the classes has no mode.

In order to generate cross-validated quantitative results, the descriptors have been randomly sampled as follows: $(10.000$ samples $\times 10$ classes $) \times 5$ folds. At each round 4 folds have been used for training the ML term in eq. $\left.1 . P\left(\bar{x}_{a} \mid \omega\right)\right)$ and the remaining fold has been used for testing. The labeling results are provided in figure 6 as boxplots representing the confusion matrices distribution across the folds. In the figure, the samples are grouped by the actual class of the sample and distributed by the predicted classes. The top label represents the samples' actual class, whereas the predicted class is color coded at the bottom. Boxplots in blue represent the results of classifying the samples using intensity, whereas the bloxpots in red represent the results obtained when using SIFT. The lack of variability within the boxplots illustrates a repeatability of the results across the samples, which gets accentuated when using SIFT. The results show that the preferred labels which cover larger portion of the feature space achieve better results than the other classes. This is more clear for the intensity case since there are classes with no mode and therefore all the samples of this class are misclassified (see fig. [5). The sensitivity or True-Positive Ratio (TPR) allows to obtain a general sense of performance across all the labels. The TPR value obtained for the intensity case is $16.6 \pm 27.5 \%$, whereas for the SIFT case is $18.8 \pm 17.2 \%$ which show that both feature spaces produce similar results. Notice 

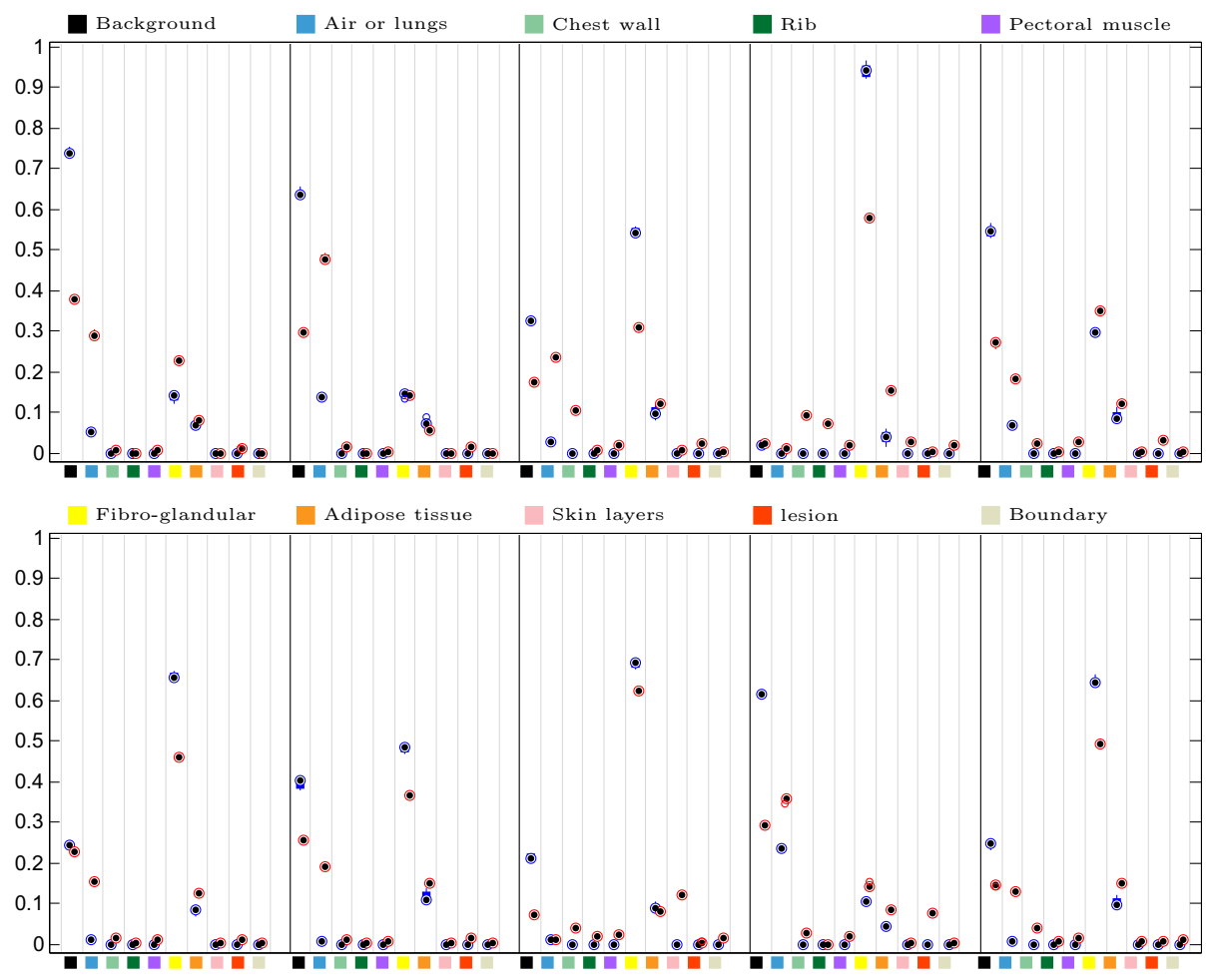

Fig. 6. Confusion matrices results distribution represented as boxplots. The results are grouped by actual class of the samples and distributed by the predicted label.

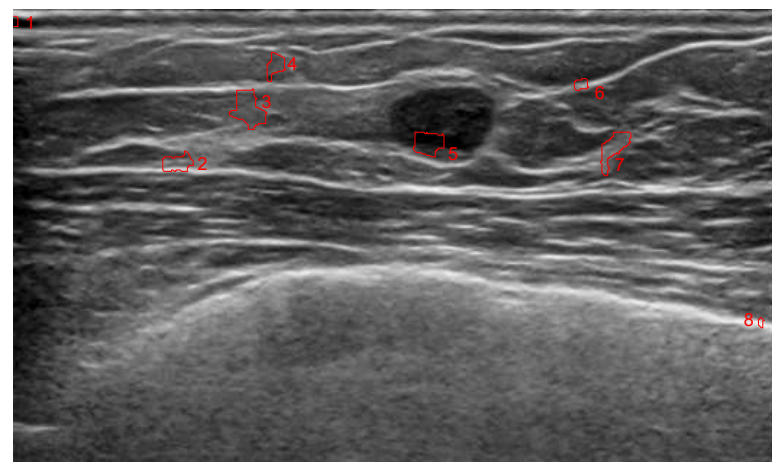

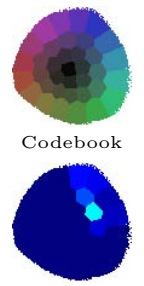

(3)

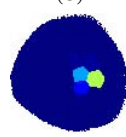

(6)

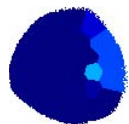

(1)

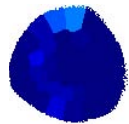

(4)

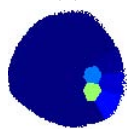

(7)

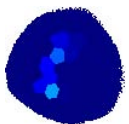

(2)

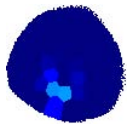

(5)

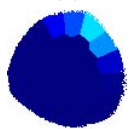

(8)

Fig. 7. SIFT-BoF descriptors qualitative analysis. (Left) image example. (Right) Dictionary representation colored using the location of the keypoint location in fig. 3 a space. (1-8) Occurrence of the dictionary's key-points associated to each region highlighted in the original image.

that the large variability reported is due to missclassification of the labels with no mode, as can be observed in figure 5 . 


\section{High-Level Texture Descriptor Using Bag-of-Features (BoF) and SIFT Descriptors}

Texture is an area property related to spatial repetition of structures, similar statistical properties of the area or both. A technique to embed statistical properties of a low level descriptor is Bag-of-Features $(\mathrm{BoF})$ which analyses the occurrence of a set of keywords (or key-points) within a particular region [2].

In our proposal, the words or features representing the images are SIFT descriptors. In order to determine the words forming the dictionary or codebook needed to generate the BoF descriptors, the space of SIFT descriptors is clustered in order to produce a hard quantification of this space. In this case, a $\mathrm{k}$-means procedure with $k=36$ is used to generate the codebook. To generate the BoF-SIFT feature, all the SIFT descriptors are substituted for the closest SIFT descriptor in the codebook. Finally the texture description from a particular area is expressed as the keywords' occurrence in this area. The descriptor is normalized so that the sum of all the occurrences is 1 .

In our application, the areas used for extracting BoF descriptors are determined by using Quick-Shift (QS) super-pixels, as is shown in figure 7 The figure shows a codebook partitioning the feature space into 36 groups along with the BoF descriptors for the 8 highlighted super-pixels. For the visualization of the BoF features a heat color coding has been used to represent the occurrence of each word within the codebook.

In order to quantitatively assess the performance of SIFT embedded within a high-level feature descriptor such as BoF, a dataset of super-pixels with its associated GT and BoF-SIFT descriptor has been build up. At this point a superpixel is eligible if it is larger than 50 pixels and is fully contained within the same GT label. This second constrain has been relaxed for skin and rib classes allowing super-pixels with $75 \%$ label contained to be eligible. The study has been carried out only for all the tissue classes, thus excluding background and boundary classes. To perform the evaluation 20 folds of 8 super-pixels (one per class) have been selected forming a set of 152 samples for training and 8 samples for testing at each round. The experiments have been repeated under the same conditions with 3 different codebooks in order to take into account the variability introduced by the codebook building. Again, for comparison purposes the experiment has been repeated using both intensity and SIFT. The classification has been carried out using Support Vector Machine (SVM). The TPR results achieved are $29 \pm$ $3.6 \%$ for the case of intensity and $33.5 \pm 2.3 \%$ for the case of SIFT, showing their similar performance and the improvement from using high-level texture descriptor over the low-level texture descriptor.

\section{Conclusions}

The present study was designed to explore the usage of SIFT feature space as a texture for characterizing the different tissues present in a breast US image. During the study, SIFT information have been used both as a low-level texture 
descriptor and encoded within a high-level texture descriptor using BoF. Performance of using the SIFT space has been evaluated by comparison with intensity. The results show that both performances are equivalent.

One of the limitaitons of this work is that all the calculations have been performed using the 2D PCA projected space which does not include all the variability of the data.

Despite these limitations, SIFT and intensity spaces produce similar results, which encourage further studies on using SIFT texture descriptors characterizing breast tissues in US images.

\section{References}

1. Cheng, H.D., Shan, J., Ju, W., Guo, Y., Zhang, L.: Automated breast cancer detection and classification using ultrasound images: A survey. Pattern Recognition 43(1), 299-317 (2009)

2. Csurka, G., Dance, C., Fan, L., Willamowski, J., Bray, C.: Visual categorization with bags of keypoints. In: Workshop on Statistical Learning in Computer Vision, ECCV, vol. 1, p. 22 (2004)

3. Jemal, A., Bray, F., Center, M.M., Ferlay, J., Ward, E., Forman, D.: Global cancer statistics. CA: A Cancer Journal for Clinicians 61(2), 69-90 (2011)

4. Lowe, D.G.: Distinctive image features from scale-invariant keypoints. International Journal of Computer Vision 60(2), 91-110 (2004)

5. Smith, R.A., Saslow, D., Sawyer, K.A., Burke, W., Costanza, M.E., Evans, W., Foster, R.S., Hendrick, E., Eyre, H.J., Sener, S.: American cancer society guidelines for breast cancer screening: Update 2003. CA: A Cancer Journal for Clinicians 53(3), 141-169 (2003)

6. Thomas Stavros, A.: Breast ultrasound. Lippincott Williams \& Wilkins (2004)

7. Yuan, Y., Giger, M.L., Li, H., Bhooshan, N., Sennett, C.A.: Multimodality computer-aided breast cancer diagnosis with ffdm and dcemri. Academic Radiology 17(9), 1158 (2010) 